EPiC Series in Engineering
Volume 3, 2018, Pages 2285-2292
HIC 2018. 13th International
Conference on Hydroinformatics

\title{
A Proposed Implicit Friction Source Term Treatment for Simulating Overland Flow
}

\author{
Tian Wang ${ }^{1,2}$, Jingming Hou ${ }^{1 *}$, Peng $\mathrm{Li}^{1}$, Jiaheng $\mathrm{Zhao}^{2}$, Ilhan Özgen ${ }^{2}$ and \\ Reinhard Hinkelmann ${ }^{2}$ \\ ${ }^{1}$ State Key Laboratory of Eco-hydraulics in Northwest Arid Region of China, Xi'an University of \\ Technology, Xi' an 710048, Shaanxi, China \\ ${ }^{2}$ Chair of Water Resources Management and Modeling of Hydrosystems, Technische Universität \\ Berlin, 13355 Berlin, Germany \\ tian.wang@wahyd.tu-berlin.de, jingming.hou@xaut.edu.cn, \\ lipeng74@163.com, jiaheng.zhao@wahyd.tu-berlin.de, \\ ilhan. oezgen@wahyd.tu-berlin. de, reinhard.hinkelmann@wahyd.tu- \\ berlin.de
}

\begin{abstract}
The fully 2D dynamic shallow water equations have been widely applied for numerical simulation of overland flow in the recent years. However, most of the existing friction term discretisation schemes do not recover the correct asymptotic flow behaviour as water depths becomes small. In this model, the shallow water equations were discretized by the framework of the Godunov-type finite volume scheme. The hydrostatic reconstruction is applied to reconstruct non-negative water depths at wetdry interfaces. Numerical fluxes are computed with a HLLC solver. The novel aspects of the model include the slope source term treatment. Specific treatment of friction source terms has been proposed to discretize the friction terms to recover the correct asymptotic behaviour of SWEs when the water depth becomes small. The accuracy and robustness of the proposed model are verified by comparing with analytical solutions. The results demonstrate that the proposed method treating friction source term is a relatively more accurate, efficient, straightforward and universal one for evaluating overland flow problems.
\end{abstract}

Keywords: Overland flow; Shallow water equations; Friction source terms; Proposed implicit; Asymptotic behavior

${ }^{*}$ Corresponding author. Tel.: +86-15809283371; fax: +86-029-83239907

E-mail address: jingming.hou@xaut.edu.cn 


\section{Introduction}

Numerical models for surface water flow, including overland and open channel flows, play an essential role in solving hydrological, hydraulic and environmental problems (Huang et al.2013; Jomaa et al.2013; Kim et al.2014). The fully dynamic shallow water equations (SWE) are generally considered to be more physically based than traditional hydrological models, due to their capability of computing flow properties in time and space (Costabile et al.2013; Kim et al.2013). One of the challenges related to solving the SWEs for overland flows is the treatment of friction source terms, which are usually expressed as a non-linear function of the flow velocity and depth, e.g. the Manning formula. The friction source terms must be well determined to account for the resistance on low water depth which is very common in overland flow.

As the water depth becomes small, the relaxation time may be much smaller than the time step determined by the Courant-Friedrichs-Lewy (CFL) condition. But using a time step bigger than the relaxation time may lead to numerical instability, causing the so-called 'stiffness problem'. Therefore, for accurate and stable simulation of overland flows, SWE model must include a proper friction term discretization scheme to remove the 'stiffness'.

Different approaches have been used in the literature to discretize the friction source terms of the SWEs (Murillo et al.2009; Liang et al.2009; Song et al.2011; Xia et al.2017). But further research effort is needed to resolve this issue for practical applications that involve simulation of overland flows over complex terrains with wetting and drying. In this paper, a proposed implicit method is applied to discretize the friction source terms of the SWEs.

\section{Governing equation}

In a vector form, the $2 \mathrm{D}$ shallow water equations can be written as:

$$
\frac{\partial \mathbf{q}}{\partial t}+\frac{\partial \mathbf{f}}{\partial x}+\frac{\partial \mathbf{g}}{\partial y}=\mathbf{S}
$$

$\mathbf{q}=\left[\begin{array}{l}h \\ q_{x} \\ q_{y}\end{array}\right], \mathbf{f}=\left[\begin{array}{c}q_{x} \\ u q_{x}+g h^{2} / 2 \\ u q_{y}\end{array}\right], \mathbf{g}=\left[\begin{array}{c}q_{y} \\ v q_{x} \\ v q_{y}+g h^{2} / 2\end{array}\right], \mathbf{S}=S_{b}+S_{f}=\left[\begin{array}{c}0 \\ -g h \partial z_{b} / \partial x \\ -g h \partial z_{b} / \partial y\end{array}\right]+\left[\begin{array}{c}0 \\ -C_{f} u \sqrt{u^{2}+v^{2}} \\ -C_{f} v \sqrt{u^{2}+v^{2}}\end{array}\right]$ (2)

where $t$ represents the time; $x$ and $y$ are the Cartesian coordinates; $\mathbf{q}$ denotes the vector of conserved flow variables consisting of $h, q_{x}$ and $q_{y}$, i.e. the water depth, unit-width discharges in $x$ - and $y$ direction, respectively; $q_{x}=u h$ and $q_{\mathrm{y}}=v h ; u$ and $v$ are depth-averaged velocities in $x$ - and $y$ directions; $\mathbf{f}$ and $\mathbf{g}$ are the flux vectors in $x$ - and $y$-direction; $\mathbf{S}$ is the source vector that may be further subdivided into slope source term $S_{b}$ and friction source term $S_{f} ; z_{b}$ represents the bed elevation; $C_{f}$ is the bed roughness coefficient that is computed by $g n^{2} / h^{1 / 3}$ with $n$ being the Manning coefficient. Moreover, the water level $\eta=h+z_{b}$ is also used in the numerical scheme adopted in this work. 


\section{Numerical schemes}

The SWEs are discretized into algebraic equations by the finite volume method. The fluxes of mass and momentum are computed by the HLLC approximate Riemann solver (i.e. Harten, Lax, and van Leer approximate Riemann solver with contact wave restored). The hydrostatic reconstruction is applied to reconstruct non-negative water depths at wet-dry interfaces. The slope source terms are evaluated by the slope flux method as proposed in (Hou et al.2013). The friction source terms are calculated by the proposed implicit method as presented in following. A two-stage explicit RungeKutta approach is applied to update the flow variables to a new time level.

To resolve state relaxation time of the friction source term much smaller than the hydrodynamic time step determined by the CFL condition for thin overland flow, a new approach is developed based on an implicit concept to deal with the friction source terms of the SWEs. It can effectively relax the flow towards the equilibrium state in a single time step and can be easily integrated into a commonly used explicit scheme.

Within an explicit framework, the unit-width discharge can be updated to a new time level $\mathrm{n}+1$ as:

$$
q^{n+1}=q^{n}+\Delta q+\Delta t S_{f}
$$

To preserve the implicit form of the friction source terms and enable the equations solvable in an analytical way, the following rearrangement is taken into account as:

$$
\begin{aligned}
& S_{f x}=-C_{f} \frac{q_{x} \sqrt{q_{x}^{2}+q_{y}^{2}}}{h^{2}}=-C_{f} \frac{q_{x}^{2}}{h^{2}} \frac{\sqrt{q_{x}^{2}+q_{y}^{2}}}{q_{x}} \\
& S_{f y}=-C_{f} \frac{q_{y} \sqrt{q_{x}^{2}+q_{y}^{2}}}{h^{2}}=-C_{f} \frac{q_{y}^{2}}{h^{2}} \frac{\sqrt{q_{x}^{2}+q_{y}^{2}}}{q_{y}}
\end{aligned}
$$

Based on the rearrangement, the assumption for linearization is employed in equation (3) through remaining the dimensional term $\sqrt{q_{x}^{2}+q_{y}^{2}} / q_{x}$ and $\sqrt{q_{x}^{2}+q_{y}^{2}} / q_{y}$ explicit as:

$$
\begin{aligned}
& q_{x}^{n+1}=q_{x}^{n}+\Delta q_{x}-\Delta t C_{f}\left(\frac{q_{x}^{n+1}}{h^{n}}\right)^{2} \frac{\sqrt{q_{x}^{n 2}+q_{y}^{n 2}}}{q_{x}^{n}} \\
& q_{y}^{n+1}=q_{y}^{n}+\Delta q_{y}-\Delta t C_{f}\left(\frac{q_{y}^{n+1}}{h^{n}}\right)^{2} \frac{\sqrt{q_{x}^{n 2}+q_{y}^{n 2}}}{q_{y}^{n}}
\end{aligned}
$$

Obviously, the equations (6) and (7) are quadratic equations with unknown variables $q_{x}^{n+1}$ and $q_{y}^{n+1}$, respectively. On the basis of the finding in last subsection, the solutions at the new time level can be derived as

$$
q_{x}^{n+1}=\frac{1-\sqrt{1+\frac{4 \Delta t C_{f}}{h^{n 2}} \frac{\sqrt{q_{x}^{n 2}+q_{y}^{n 2}}}{q_{x}^{n}+\theta}\left(q_{x}^{n}+\Delta q_{x}\right)}}{\left(-\frac{2 \Delta t C_{f}}{h^{n 2}} \frac{\sqrt{q_{x}^{n 2}+q_{y}^{n 2}}}{q_{x}^{n}+\theta}\right)}
$$


$q_{y}^{n+1}=\frac{1-\sqrt{1+\frac{4 \Delta t C_{f}}{h^{n 2}} \frac{\sqrt{q_{x}^{n 2}+q_{y}^{n 2}}}{q_{y}^{n}}\left(q_{y}^{n}+\Delta q_{y}\right)}}{\left(-\frac{2 \Delta t C_{f}}{h^{n 2}} \frac{\sqrt{q_{x}^{n 2}+q_{y}^{n 2}}}{q_{y}^{n}+\theta}\right)}$

$\theta=1.0 e^{-12}$ is used to avoid dividing by zero. Obviously, for $1 \mathrm{D}$ case i.e. $q_{y}=\mathbf{0}$, becomes:

$$
q_{x}^{n+1}=\frac{1-\sqrt{1+\frac{4 \Delta t C_{f}}{h^{n 2}} \frac{\left|q_{x}^{n}\right|}{q_{x}^{n}+\theta}\left(q_{x}^{n}+\Delta q_{x}\right)}}{\left(-\frac{2 \Delta t C_{f}}{h^{n 2}} \frac{\left|q_{x}^{n}\right|}{q_{x}^{n}+\theta}\right)}
$$

\section{Results and discussion}

\subsection{A theoretic test in various overland flow condition}

To demonstrate the performance of the proposed method treating friction source term under various overland flow conditions with relatively thin water, a theoretic case is devised in a $1 \mathrm{D}$ channel with different water depths, velocities and slopes and categorized by A, B, C and D as shown in Table 1. In this benchmark test, the updated flow variables should be in an asymptotic behavior to the uniform flow condition as a constant slope and friction coefficient along the channel, i.e. if the flow velocity is lower than the uniform one under the same unit-width discharge, acceleration will take place until the velocity reaches the uniform one, and vice versa. In a computational time step, the updated $q^{n+1}$ should become closer or equal to the unit-width equilibrium discharge $q^{e}$ when compared to $q^{n}$. It is therefore lead to $\Delta q$ computed from equation (11) to be $0 \mathrm{~m}^{2} / \mathrm{s}$, if the numerical scheme is in an asymptotic behavior, otherwise, a non-zero value of $\Delta q$ will be produced for a method failing to handle the friction source term treatment for thin overland flow.

$$
\Delta q=\left|q^{n+1}-q^{n}\right|+\left|q^{n+1}-q^{e}\right|-\left|q^{e}-q^{n}\right|
$$

In this test case, a widely used approach discretizing the friction source terms of the SWEs developed and applied in Hou et al. (2013) and termed as splitting point-implicit method, is used as a reference.

Table 1 Thin water flow benchmark test under different water depth, velocity and slope conditions

\begin{tabular}{cccccccc}
\hline Category & Case & $h(\mathrm{~m})$ & $\begin{array}{c}n \\
\left(\mathrm{~s} / \mathrm{m}^{1 / 3}\right)\end{array}$ & $u(\mathrm{~m} / \mathrm{s})$ & $d x(\mathrm{~m})$ & Courant & $\begin{array}{c}i \\
(\%)\end{array}$ \\
\hline \multirow{4}{*}{$\mathrm{A}$} & 1 & 0.004 & 0.01 & 0.65 & 2 & 0.1 & 5 \\
& 2 & 0.0035 & 0.01 & 0.65 & 2 & 0.1 & 5 \\
& 3 & 0.003 & 0.01 & 0.65 & 2 & 0.1 & 5 \\
& 4 & 0.0025 & 0.01 & 0.65 & 2 & 0.1 & 5 \\
& 5 & 0.002 & 0.01 & 0.65 & 2 & 0.1 & 5 \\
& 6 & 0.0015 & 0.01 & 0.65 & 2 & 0.1 & 5 \\
\hline
\end{tabular}




\begin{tabular}{cccccccc}
\hline & 7 & 0.001 & 0.01 & 0.65 & 2 & 0.1 & 5 \\
& 1 & 0.004 & 0.01 & 0.65 & 2 & 0.02 & 5 \\
& 2 & 0.0035 & 0.01 & 0.65 & 2 & 0.02 & 5 \\
& 3 & 0.003 & 0.01 & 0.65 & 2 & 0.02 & 5 \\
& 4 & 0.0025 & 0.01 & 0.65 & 2 & 0.02 & 5 \\
& 5 & 0.002 & 0.01 & 0.65 & 2 & 0.02 & 5 \\
& 6 & 0.0015 & 0.01 & 0.65 & 2 & 0.02 & 5 \\
& 7 & 0.001 & 0.01 & 0.65 & 2 & 0.02 & 5 \\
& 1 & 0.001 & 0.01 & 0.65 & 2 & 0.1 & 5 \\
& 2 & 0.001 & 0.01 & 0.55 & 2 & 0.1 & 5 \\
& 3 & 0.001 & 0.01 & 0.45 & 2 & 0.1 & 5 \\
& 4 & 0.001 & 0.01 & 0.35 & 2 & 0.1 & 5 \\
& 5 & 0.001 & 0.01 & 0.25 & 2 & 0.1 & 5 \\
& 6 & 0.001 & 0.01 & 0.15 & 2 & 0.1 & 5 \\
& 7 & 0.001 & 0.01 & 0.05 & 2 & 0.1 & 5 \\
& 1 & 0.001 & 0.01 & 0.25 & 2 & 0.1 & 50 \\
& 2 & 0.001 & 0.01 & 0.25 & 2 & 0.1 & 25 \\
& 3 & 0.001 & 0.01 & 0.25 & 2 & 0.1 & 10 \\
& 3 & 0.001 & 0.01 & 0.25 & 2 & 0.1 & 5 \\
& 4 & 0.001 & 0.01 & 0.25 & 2 & 0.1 & 0.25 \\
& 5 & 0.001 & 0.01 & 0.25 & 2 & 0.1 & 0.1 \\
& 6 & 0.001 & 0.01 & 0.25 & 2 & 0.1 & 0.5 \\
\hline
\end{tabular}

Note: $h$ is depth of water; $n$ is Manning coefficient; $u$ is velocity of flow; $d x$ is the grid size; $i$ is the channel slope.

Category A focuses on the effects of varying water depth on the friction source terms. The computed results indicate that the propose $\mathrm{D}$ approach is able to produce the asymptotic results to an equilibrium flow state, reflected by $\Delta q$ remain $0 \mathrm{~m}^{2} / \mathrm{s}$ for all water depths considered (Figure 1a). This behavior cannot be maintained by the existing approach, as the computed $\Delta q$ is greater than 0 $\mathrm{m}^{2} / \mathrm{s}$, when the water depth were below $0.0025 \mathrm{~m}$, indicating the existing approach will lead to unrealistic evaluation of the friction source term when the water depth is smaller than certain value.

Category B is mainly concerned with the effects of the computational time step on the friction source terms, on the basis of the category B. In this case, the Courant number is set to be 0.02 which is $1 / 5$ times smaller than that in the category $\mathrm{A}$, aiming at decreasing the side effect of the unrealistic friction. The results depict that all of the $\Delta q$ are equal to $0 \mathrm{~m}^{2} / \mathrm{s}$ (Figure 1b), indicating that the overland flow with small water depth could be well reproduced for much smaller time step using either the existing or proposed treatment for friction source terms. But the considerably reduced time step will in turn increase the computation time.

Category $\mathrm{C}$ is devised to demonstrate the influence of the velocity on thin water flow condition with the value under $0.001 \mathrm{~m}$, which is likely to cause computation failure as shown in Fig. 1a. The initial condition for the flow is imposed by a constant water depth of $0.001 \mathrm{~m}$ and the velocities of $0.65,0.55,0.45,0.35,0.25,0.15,0.05 \mathrm{~m} / \mathrm{s}$ (Figure 1c), respectively. The results show that $\Delta q$ values 
are greater than $0 \mathrm{~m}^{2} / \mathrm{s}$ under different velocities with the existing approach, indicating it fails to handle the overland flow problem with lower water depth. On the contrary, the $\Delta q$ computed by the proposed approach is equal to $0 \mathrm{~m}^{2} / \mathrm{s}$ under any velocity, expressing the numerical solution is in an asymptotic behavior.
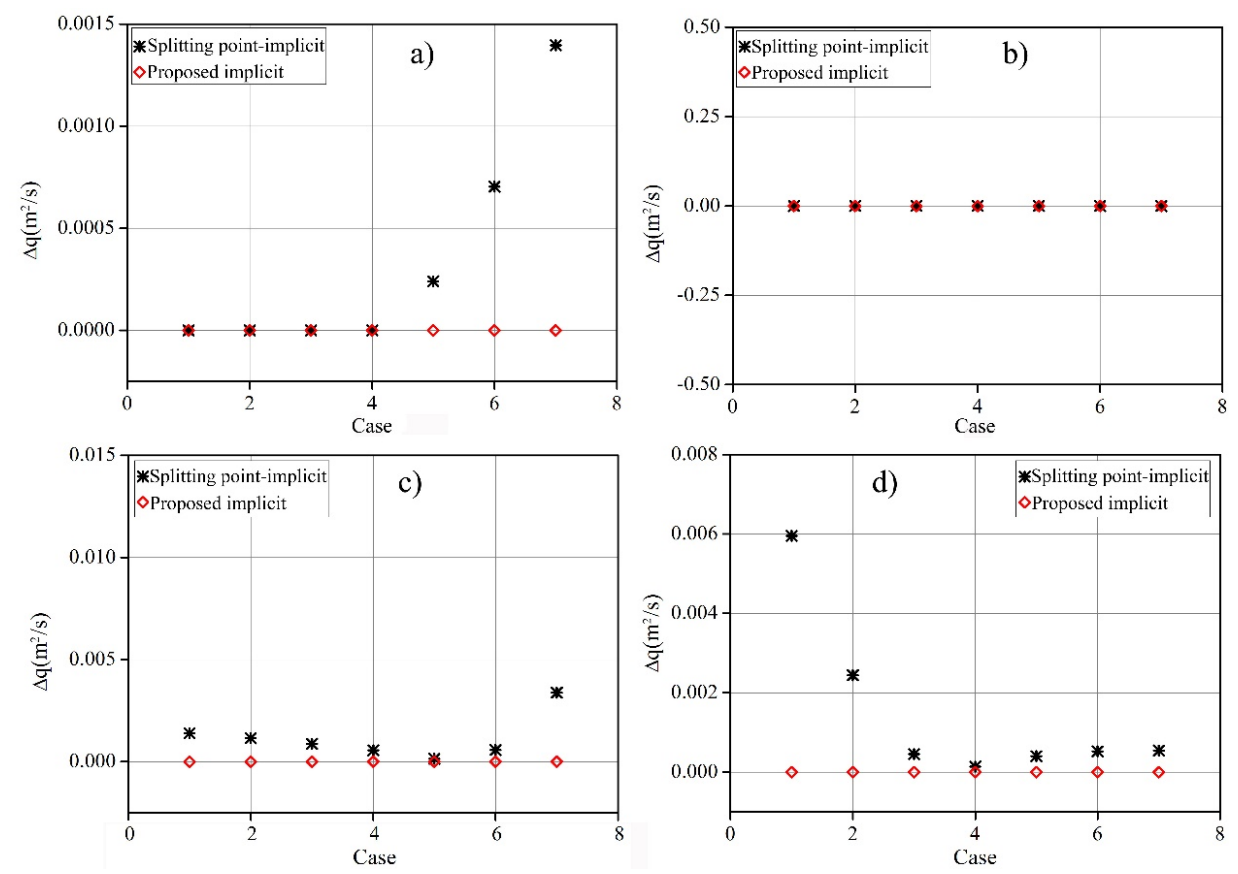

Figure 1: values in different kinds under explicit and improved implicit approaches

Category D is applied herein to analyze the effects of slope channel on the overland flow simulation. When using the selected existing approach, the results show that $\Delta q$ are greater than 0 $\mathrm{m}^{2} / \mathrm{s}$ on seven slopes (Figure 1d), demonstrating the existing method could not produce reliable simulation for overland flow of thin water depth. For the proposed method, the performance achieving the equilibrium flow state can be preserved, which is illustrated by $\Delta q$ equal to 0 for all slopes under consideration.

\subsection{An asymptotic process simulation for evaluating computational efficiency}

This test case is devised to compare the computational efficiency between the proposed method and the fully implicit one using the criteria suggested in (Xia et al. 2017), when evaluating the friction source term. As sketched in Fig.1, on a fixed slope, given inflow and free outflow conditions are set up at the up and downstream boundaries, respectively. A set of inflow conditions are defined with the water depth and velocity of $0.1 \mathrm{~m}$ and $2.41 \mathrm{~m} / \mathrm{s}, 0.04 \mathrm{~m}$ and $1.37 \mathrm{~m} / \mathrm{s}, 0.01 \mathrm{~m}$ and $0.52 \mathrm{~m} / \mathrm{s}$, respectively. The friction calculation efficiency is focused for the asymptotic behavior process, i.e. the flow status varying from non-uniform flow to uniform one. The friction source term calculation iterates 6 to 8 times by using the full implicit approach for the asymptotic behavior process. Compared to the fully implicit approach, the proposed method could save $45.16 \%$ to $93.55 \%$ computational time for simulating the asymptotic behavior process. It should be noted that, the iteration time will reduce to 2 when the flow becomes uniform or quasi-uniform for the fully implicit 
approach. In this case, the high efficiency of the proposed approach is not obvious, however, it is still relatively superior for easy implementation for coding, due to an explicit and straightforward form.

\subsection{Overland flow modelling in a V-shaped catchment}

This test case simulated rainfall-runoff in a V-shaped catchment. An approximate analytical solution was found for the V-shaped schematic catchment by Overton and Brakensiek (1973), which is illustrated in Figure 2. The simulations were made on the basis of a test case of a double slope on the plane and a single slope in the channel (Figure 2). The slope of the two hillsides is 0.05 , and the channel slope is 0.02 . The channel has a width of $20 \mathrm{~m}$ and a length of $1000 \mathrm{~m}$.

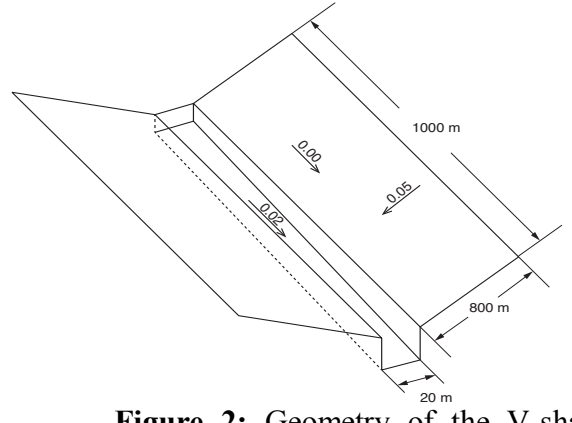

Figure 2: Geometry of the V-shaped

The simulation was conducted using a rainfall input with an intensity of $10.8 \mathrm{~mm} / \mathrm{h}$ and a duration of $1.5 \mathrm{~h}$. The domain was discretized by a uniform rectangular mesh with a cell size of $5 \mathrm{~m}$. The CFL criterion was set to 0.5 . Values for the Manning's coefficients of $n=0.015 \mathrm{~s} / \mathrm{m}^{1 / 3}$ for the hillsides and $\mathrm{n}=0.15 \mathrm{~s} / \mathrm{m}^{1 / 3}$ for the channel were assigned which, although unrealistic in natural conditions, were assumed so as to obtain a similar characteristic concentration time both in the hillsides and in the channel. All boundaries beside the channel flow are closed boundaries, except the outlet, where critical flow depth was assumed. Splitting implicit and full implicit methods for the friction source term treatment proposed by Liang et al. (2009) and Xia et al. (2017) were compared with the proposed implicit method, respectively.

Figure 3 plots the hydrographs at the hillsides and channel outlet predicted by the four different models (i.e. proposed implicit; splitting point-implicit; explicit; fully implicit), comparing with the analytical solutions derived based on the kinematic wave assumption (Di Giammarco et al. 1994). The hydrographs predicted by the proposed implicit and fully implicit dealing with the friction source terms, i.e. the model developed in this work, agree closely with the analytical solutions. However, the other two models failed to produce correct hydrographs at both the hillsides and channel outlet by the splitting point implicit and explicit methods. Therefore, the proposed implicit method proposed by the authors was the most positive and effective method.
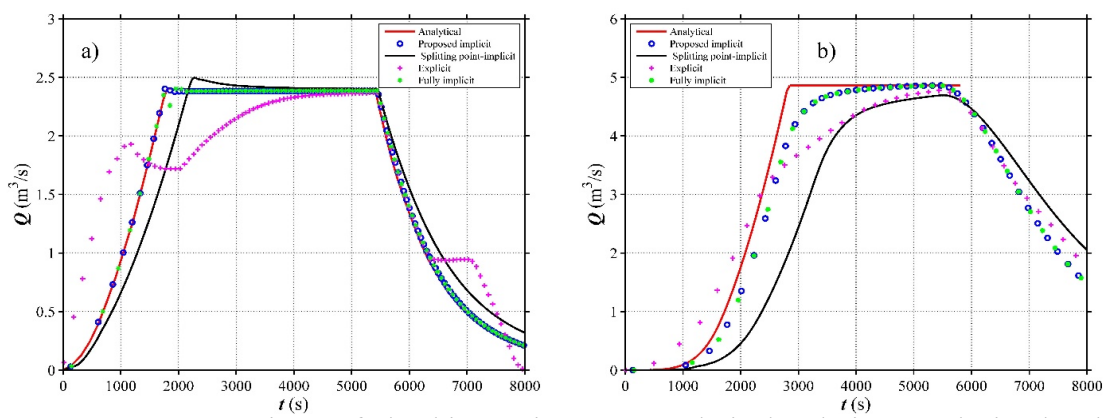

Figure 3: Comparison of the kinematic wave analytical solutions and simulated hydrographs at the hillside (a) and channel outlet (b) 


\section{Conclusion}

In this paper, a proposed implicit method is used to discretize the friction terms to recover the correct asymptotic behaviour of SWEs when the water depth becomes small. The model was verified by an idealized analytical test case. Four treatments of friction terms were carried out to simulate the runoff in the V-shaped schematic catchment. The results demonstrate that the proposed method treating friction source term is a relatively more accurate, efficient, straightforward and universal one for evaluating overland flow problems.

\section{Acknowledgement}

This work is partly supported by the National Key R\&D Program of China (No.2016YFC0402404, 2016YFC0402704); National Natural Science Foundation of China (No.19672016); the School foundation of Xi'an University of Technology (No. 310-252071711); China Scholarship Council (CSC) who supports this research is gratefully acknowledged.

\section{Reference}

Costabile, P., Costanzo, C., \& Macchione, F. (2013). A storm event watershed model for surface runoff based on $2 \mathrm{~d}$ fully dynamic wave equations. Hydrological Processes.27, 554-569.

Di Giammarco, P., Todini, E., Consuegra, D., Joerin, F. and Vitalini, F. (1994). Combining a 2-D flood plain model with GIS for flood delineation and damage assessment. In: P. Molinaro and L. Natale (Editors), Modelling of Flood Propagation over Initially Dry Areas, Proc. Specialty Conf. ENEL-DSRCRIS, Milan. ASCE, New York, pp. 171-185.

Huang, P. C., \& Lee, K. T. (2013). An efficient method for DEM-based overland flow routing. Journal of Hydrology. 489, 238-245.

Hou, J., Liang, Q., Simons, F., \& Hinkelmann, R. (2013). A stable 2d unstructured shallow flow model for simulations of wetting and drying over rough terrains. Computers \& Fluids.82,132-147.

Jomaa, S., Barry, D. A., Heng, B. C. P., Brovelli, A., Sander, G. C., \& Parlange, J. Y. (2013). Effect of antecedent conditions and fixed rock fragment coverage on soil erosion dynamics through multiple rainfall events. Journal of Hydrology.484, 115-127.

Kim, D. H., \& Seo, Y. (2013). Hydrodynamic analysis of storm movement effects on runoff hydrographs and loop-rating curves of a v-shaped watershed. Water Resources Research.49, 6613-6623.

Kim, J., \& Ivanov, V. Y. (2014) On the no uniqueness of sediment yield at the catchment scale: the effects of soil antecedent conditions and surface shield. Water Resources Research.50, 1025-1045.

Liang, Q., \& Marche, F. (2009) Numerical resolution of well-balanced shallow water equations with complex source terms. Advances in Water Resources.32, 873-884.

Murillo, J., García-Navarro, P., \& Burguete, J. (2009). Time step restrictions for well-balanced shallow water solutions in non-zero velocity steady states. International journal for numerical methods in fluids.60, 1351-1377.

Overton, D.E. and Brakensiek, D.L. (1973). A kinematic model of surface runoff response. IAHS Publ.,96, 110-112.

Song, L., Zhou, J., Li, Q., Yang, X., \& Zhang, Y. (2011). An unstructured finite volume model for dam-break floods with wet/dry fronts over complex topography. International Journal for Numerical Methods in Fluids.67, 960-980.

Xia, X., Q. Liang, X. Ming, \& Hou, J. (2017). An efficient and stable hydrodynamic model with novel source term discretization schemes for overland flow and flood simulations, Water Resour. Res. 53, 3730-3759. 